\title{
SMALL MODULES
}

W. W. LEONARD

Introduction. In [1] and [5] a left $A$-module $E$ is said to be small (or superfluous) in $F$ if $E+H=F$ for any submodule $H$ of $F$ implies $H=F$. We define a left $A$-module $S$ to be small if it is a small submodule of some module. In what follows we investigate some properties of small modules and prove the following theorems:

Theorem. A torsion module over a principal ideal domain is small if and only if the primary components are bounded.

Theorem. If $A$ is a discrete valuation ring with prime $p$, and $G$ an $A$-module then the following conditions are equivalent:

(1) $G$ is small,

(2) $p G$ is small in $G$,

(3) $G$ is the direct sum of a free module of finite rank and a bounded torsion module.

The notation used in the following will be that of [2] and [3]. ${ }^{1}$

Lemma 1. If $E, F$, and $G$ are left $A$-modules such that $E \subset F \subset G$ and $E$ is small in $F$ then $E$ is small in $G$.

Proof. Straightforward.

Lemma 2. If $S$ is a small submodule of a left $A$-module $F$ and $S$ is contained in a direct summand $E$ of $F$ then $S$ is small in $E$.

Proof. Straightforward.

Theorem 1. A left $A$-module $F$ is small if and only if $F$ is small in its injective envelope.

Proof. We will denote the injective envelope of a module $F$ by $I(F)$.

If $F$ is small in $I(F)$ then $F$ is a small module by definition. Thus, suppose $F$ is a small submodule of a left $A$-module $H$. Then $F \subset H$ $C I(H)$, so by Lemma $1 F$ is small in $I(H)$. Assume $F+G=I(F)$ for some submodule $G$ of $I(F)$. Since $I(F)$ is injective it is a direct summand of $I(H)$ and $F \subset I(F)$. Thus, by Lemma $2 F$ is small in $I(F)$.

Received by the editors July 9,1965 .

1 The author is indebted to Dr. E. E. Enochs for his help and suggestions. He is also obliged to the referee for his suggestions, especially for the proof 1 implies 3 of Theorem 5. 
TheOREM 2. Submodules, quotient modules and finite direct sums of small modules are small.

Proof. Straightforward.

Corollary. The finite sum of small left $A$-modules which are submodules of a given module is a small module.

If $I$ is an infinite set it can be shown that $Z^{(I)}$ is not small in $Q^{(I)}$ and $Z^{N}$ is not small in $Q^{N}$ where $Z$ is the additive group of integers, $Q$ the additive group of rational numbers, and $N$ the set of positive integers. But, $Z$ is a small group. Moreover, $Z\left(p^{\infty}\right)$ is the sum of all its proper subgroups, their injective envelope, and each subgroup is small in $Z\left(p^{\infty}\right)$, but $Z\left(p^{\infty}\right)$ is not small.

We now show that a module over a principal ideal domain is small if and only if its torsion and torsion free parts are small.

Lemma 3. If $E$ is a left $A$-module and $S \subset F$ are submodules of $E$ such that $S$ is small in $E$ then $F / S$ is small in $E / S$ if and only if $F$ is small in $E$.

Proof. Suppose $F+H=E$ for some submodule $H$ of $E$. Then $F / S=(H+S) / S=E / S$, but $F / S$ is small in $E / S$, hence $(H+S) / S$ $=E / S$. Therefore, $H+S=E$. But, $S$ is small in $E$, hence $H=E$. Thus $F$ is small in $E$.

Conversely, assume $F / S+H / S=E / S$ for some submodule $H$ containing $S$ of $E$. Then $(F+H) / S=E / S$, hence $F+H=E$. But, $F$ is small in $E$, hence $H=E$. Thus, $F / S$ is small in $E / S$.

THEOREM 3. If $A$ is a left hereditary ring and the sequence of left $A$-modules, $0 \rightarrow H \rightarrow G \rightarrow G / H \rightarrow 0$, is exact then $H$ and $G / H$ are small modules if and only if $G$ is a small module.

Proof. Since $H$ is small in $I(H), H$ is small in $I(G)$. Moreover, $G / H$ is small in $I(G) / H$ since $I(G) / H$ is injective for $A$ a hereditary ring. Hence, by Lemma $3 G$ is small in $I(G)$, therefore a small module.

Conversely, if $G$ is small then $H$ is small and $G / H$ is a small module by Theorem 2 .

Corollary. A module over a principal ideal domain is small if and only if its torsion and torsion free parts are small.

Proof. A principal ideal domain is a hereditary ring.

Lemma 4. If $G$ is a small module over a principal ideal domain then the torsion submodule, $T(G)$, is the only basic submodule of itself. 
Proof. $G$ is small in $I(G)$, hence $T(G)$ is small in $I(G)$. If $B$ is a basic submodule of $T(G)$ then $T(G) / B$ is small in $I(G) / B$. But, $T(G) / B$ is divisible, hence $T(G) / B=0$ or $T(G)=B$.

Lemma 5 (Kulikov). A primary module over a principal ideal domain has only one basic subgroup if and only if it is either divisible or bounded.

Proof. [3, Theorem 31.3].

Theorem 4. A torsion module $T$ over a principal ideal domain is small if and only if the primary components of $T$ are bounded.

Proof. Suppose $T$ is small. $T$ has a unique decomposition into its primary components and by Lemma $4 T$ is the only basic submodule of itself. Since $T$ is not divisible by Lemma 5 the primary components are bounded.

Conversely, assume the primary components of $T$ are bounded. Suppose $T+H=I(T)$ for some submodule $H$ of $I(T)$. Then $T_{p}+H_{p}$ $=I(T)_{p}$ where $T_{p}, H_{p}$, and $I(T)_{p}$ are the respective primary components. There exists an integer $N>0$ such that $p^{N} T_{p}=0$. Hence, $p^{N}\left(T_{p}+H_{p}\right)=p^{N} H_{p}=p^{N} I(T)_{p}=I(T)_{p}$. Then $H=\oplus_{p} H_{p}=\oplus_{p} I(T)_{p}$ $=\oplus_{p} I\left(T_{p}\right)=I(T)$. Therefore, $T$ is a small module.

Lemma 6. If $A$ is a left hereditary ring then a left $A$-module $F$ is small if and only if $F$ has no nontrivial injective quotients.

Proof. Assume $F$ is not a small module. Then there exists a submodule $H$ or $I(F)$ such that $F+H=I(F)$ and $H \neq I(F)$. Then the sequence $0 \rightarrow F \cap H \rightarrow F \rightarrow I(F) / H \rightarrow 0$ is exact and $I(F) / H$ is injective.

Conversely, if $F / H \neq 0$ is injective for some submodule $H$ of $F$ then $F / H$ is a direct summand of $I(F) / H$. Thus, by Lemma $3 F$ is not small in $I(F)$.

Lemma 7. A small torsion free module over a principal ideal domain $A$ has finite rank.

Proof. Suppose $G$ is small, $r k(G)=\infty$, and $\left(x_{i}\right)_{i \in N}$ is a maximal linearly independent family of $G$. If $K$ is the submodule generated by $\left(x_{i}\right)_{i \in N}$ then $K$ is isomorphic to $A^{(N)}$ which is not small. Therefore, $G$ is not small; contradiction.

If $A$ is a principal ideal domain $A_{p}$ will denote the localization of $A$ at the prime $p$ and $G_{p}=A_{p} \otimes_{A} G$ the localization of the $A$-module $G$.

Lemma 8. If $A$ is a principal ideal domain and $G$ an $A$-module then $G$ is small if and only if $G_{p}$ is small for all primes $p$. 
Proof. Suppose $G_{p}$ is small for all primes $p$ and let $J$ be an injective quotient of $G$. Then the exactness of the sequence $G \rightarrow J \rightarrow 0$ implies the sequence $G_{p} \rightarrow J_{p} \rightarrow 0$ is exact for every prime $p$. Moreover, $J_{p}$ is injective. But, $J_{p}=0$ for every prime $p$ if and only if $J=0$ ([2], p. 82). Hence, the conclusion follows from Lemma 6 . (Note that this proof holds for any commutative ring.)

Suppose that $G$ is small and $J=G_{p} / H \neq 0$ is an injective quotient. We may take $J$ to be torsion. There is an $E \subset G$ such that $H=E_{p}$, and we have $J=G_{p} / E_{p}=(G / E)_{p}$. It follows that $G / E$ is torsion with p-primary component $J$, so $J$ is a quotient of $G$. Since $J$ is automatically $A$-injective $G$ is not small; contradiction.

THEOREM 5. Let $A$ be a discrete valuation ring with prime $p$, and let $G$ be an $A$-module. The following conditions are equivalent:

(1) $G$ is small,

(2) $p G$ is small in $G$,

(3) $G$ is the direct sum of a free module of finite rank and a bounded torsion module.

Proof. If $H \subset G$ then $G / H$ is injective $\Leftrightarrow p(G / H)=G / H \Leftrightarrow H+p G$ $=G$. Thus, $(1) \Leftrightarrow(2)$ follows from Lemma 6 .

$(3) \Rightarrow(1)$ follows from Theorem 2 , Theorem 4 , and the fact that $A$ is small.

$(1) \Rightarrow(3)$. By Theorem 4 the torsion part of $G$ is bounded. Using the corollary to Theorem 6 we reduce the problem to the torsion free case. By Lemma $7 G$ then has finite rank. We want to show that $G$ is finitely generated, so suppose it is not. Choose $F$ a free submodule of $G$ such that $G / F$ is torsion. $G / F$ is not finitely generated since $F$ is and $G$ is not. Let $\hat{A}$ denote the completion of $A$. By Theorem 20 [4] $\hat{A} \otimes G$ is the direct sum of a free and a divisible module. Since $G / F$ $=\hat{A} \otimes(G / F)=(\hat{A} \otimes G) /(\hat{A} \otimes F)$ is not finitely generated neither is $\hat{A} \otimes G$. Hence, $\hat{A} \otimes G$ has a nontrivial divisible part so, since $\hat{A} \otimes F$ is free, it follows that $G / F$ contains a nontrivial divisible module. This contradicts the assumed smallness of $G$.

Corollary. If $A$ is a principal ideal domain and if $G$ is an $A$ module then $G$ is small if and only if $G$ is locally a free module of finite rank plus a bounded torsion module.

\section{REFERENCES}

1. H. Bass, Finistic dimension and a homological generalization of semi-primary rings, Trans. Amer. Math. Soc. 95 (1960) 466-488.

2. N. Bourbaki, Algèbre commutative, Chapitre II, Hermann, Paris, 1960.

3. L. Fuchs, Abelian groups, Pergamon Press, New York, 1960. 
4. I. Kaplansky, Infinite abelian groups, Univ. of Michigan Press, Ann Arbor, Mich., 1954.

5. E. Mares, Semi-perfect modules, Math. Z. 82 (1963) 300.

Georgia State College

\section{AN ADDITION TO ADO'S THEOREM'1}

\section{G. HOCHSCHILD}

The main purpose of this note is to point out the following strengthened (with respect to the nilpotency property) form of the theorem on the existence of a faithful finite-dimensional representation of a finite-dimensional Lie algebra.

TheOREM 1. Let $L$ be a finite-dimensional Lie algebra over an arbitrary field, and let $\alpha$ denote the adjoint representation of $L$. There exists a faithful finite-dimensional representation $\rho$ of $L$ such that $\rho(x)$ is nilpotent for every element $x$ of $L$ for which $\alpha(x)$ is nilpotent.

For the suggestion that this nilpotency property of $\rho$ might be secured I am indebted to Leonard Ross who used the characteristic 0 case of Theorem 1 in his proof of Ado's Theorem for graded Lie algebras (Thesis, Cohomology of graded lie algebras, University of California, Berkeley, 1964).

In the case of characteristic 0 , it is known that there exists a faithful finite-dimensional representation of $L$ whose restriction to the maximum nilpotent ideal of $L$ is nilpotent [1, pp. 202-203]. Hence, in order to establish Theorem 1 in the case of characteristic 0 , it suffices to make the following observation:

Let $L$ be a finite-dimensional Lie algebra over a field of characteristic 0 , and let $M$ be a finite-dimensional L-module on which the maximum nilpotent ideal $N$ of $L$ is nilpotent. Let $x$ be an element of $L$ whose adjoint image $\alpha(x)$ is nilpotent. Then $x$ is nilpotent on $M$.

Proof. Write $L=S+R$, where $R$ is the radical of $L$ and $S$ is a semisimple subalgebra of $L$. Accordingly, write $x=s+r$, with $s$ in $S$ and $r$ in $R$. Since $\alpha(x)$ is nilpotent, it is clear that the adjoint representation of $S$ sends $s$ onto a nilpotent derivation of $S$. Since $S$

Received by the editors July 23, 1965.

1 Research done with support of NSF Grant GP 1610. 